\title{
Laboratory identification and clinical characteristics of Streptococcus bovis/Streptococcus equinus complex bacteremia: a retrospective, multicenter study in Hiroshima, Japan
}

Yuki Kaiki ${ }^{1}$, Hiroki Kitagawa ${ }^{1,2,3^{*}}$, Kayoko Tadera ${ }^{3,4,5}$, Hiroyuki Taogoshi, ${ }^{1,6}$, Mitsuyasu Ikeda 3,7, Mikihiro Kano 1,8, Toshie Harino ${ }^{9}$, Toshihito Nomura ${ }^{2}$, Keitaro Omori ${ }^{2}$, Norifumi Shigemoto ${ }^{1,2,3,10}$, Shinya Takahashi ${ }^{1}$ and Hiroki Ohge $\mathrm{e}^{2,3}$

\begin{abstract}
Background: Bacteremia due to the Streptococcus bovis/Streptococcus equinus complex (SBSEC) is associated with specific diseases, such as colorectal cancer and infective endocarditis. This study aimed to evaluate the clinical characteristics of SBSEC bacteremia and the accuracy of identification of matrix-assisted laser desorption/ionization time-offlight mass spectrometry (MALDI-TOF MS) and phenotypic identification systems for SBSEC isolates.

Methods: We analyzed patients with SBSEC bacteremia retrospectively between 2012 and 2019 at three hospitals in Japan. We re-identified each SBSEC isolate using sequencing superoxide dismutase (sodA) analysis, MALDI-TOF MS using the MALDI Biotyper, and phenotypic identification using the VITEK2.

Results: During the study period, 39 patients with SBSEC bacteremia were identified. S. gallolyticus subsp. pasteurianus (SGSP, $n=29$ ), S. gallolyticus subsp. gallolyticus (SGSG, $n=5)$, S. lutetiensis (SL, $n=4)$, and S. infantarius subsp. infantarius $(n=1)$ were identified using sodA sequencing analysis. Primary bacteremia (36\%) was the most common cause of bacteremia, followed by infective endocarditis (26\%) and biliary tract infections (23\%). Colorectal cancer was associated significantly with SGSG bacteremia, while the sources of bacteremia were similar in each SBSEC subspecies. The MALDI Biotyper was significantly more accurate in identifying the SBSEC isolates at the subspecies level compared to the VITEK2 (92\% vs. 67\%, $P=0.010$ ). In contrast, there were no significant differences in the rates of correct identification of the SBSEC isolates at the species level between the MALDI Biotyper and the VITEK2 (100\% vs. 87\%, $P=0.055)$.
\end{abstract}

Conclusions: Bacteremia with SGSG was associated with colorectal cancer, and the sources of bacteremia were similar in each SBSEC subspecies. The MALDI-TOF MS was significantly more accurate in identifying SBSEC isolates at the subspecies level than the phenotypic identification systems. The accurate identification of SBSEC isolates using the MALDI-TOF MS and phenotypic identification systems was sufficient at the species level, but it was insufficient at

\footnotetext{
*Correspondence: hkitaga@hiroshima-u.ac.jp

${ }^{1}$ Department of Surgery, Graduate School of Biomedical and Health

Sciences, Hiroshima University, Hiroshima, Japan

Full list of author information is available at the end of the article
}

(c) The Author(s) 2021. Open Access This article is licensed under a Creative Commons Attribution 4.0 International License, which permits use, sharing, adaptation, distribution and reproduction in any medium or format, as long as you give appropriate credit to the original author(s) and the source, provide a link to the Creative Commons licence, and indicate if changes were made. The images or other third party material in this article are included in the article's Creative Commons licence, unless indicated otherwise in a credit line to the material. If material is not included in the article's Creative Commons licence and your intended use is not permitted by statutory regulation or exceeds the permitted use, you will need to obtain permission directly from the copyright holder. To view a copy of this licence, visit http://creativecommons.org/licenses/by/4.0/. The Creative Commons Public Domain Dedication waiver (http://creativeco mmons.org/publicdomain/zero/1.0/) applies to the data made available in this article, unless otherwise stated in a credit line to the data. 
the subspecies level. Therefore, it may be reasonable for clinicians to perform echocardiographies and colonoscopies in all patients with SBSEC bacteremia.

Keywords: Streptococcus bovis, Bloodstream infection, MALDI-TOF MS, sodA, Colorectal cancer, Infective endocarditis

\section{Background}

Streptococcus bovis/Streptococcus equinus complex (SBSEC) is common inhabitants of the digestive tract in animals and humans. The introduction of new nomenclature of SBSEC and development of molecular techniques for identification have revealed specific disease associations with different SBSEC species and subspecies [1-3]. Streptococcus gallolyticus subsp. gallolyticus (SGSG) bacteremia is associated with infective endocarditis and colorectal cancer [4, 5]. Streptococcus gallolyticus subsp. pasteurianus (SGSP) and Streptococcus infantarius are associated with biliary tract infections and biliary-pancreatic cancer [6-9]. Therefore, an accurate identification of SBSEC at the subspecies level may be required. The most reliable methods currently available for identifying SBSEC species are gene-based methods, such as partial sequences of $\operatorname{sod} A$, gyrB, and $\operatorname{groE}[10]$. However, phenotypic identification systems remain standard in clinical laboratories. Matrix-assisted laser desorption/ionizationtime of flight mass spectrometry (MALDI-TOF MS) has been used widely as an alternative for bacterial identification in clinical microbiology and infectious disease fields. However, only a few studies have evaluated the performance of MALDI-TOF MS in the accurate identification of the species and subspecies of SBSEC [11-13]. Additionally, SBSEC infections are limited and have not been investigated fully $[14,15]$. Therefore, this study aimed to identify the clinical characteristics of SBSEC bacteremia in Japanese patients and evaluate the accuracy of identification of the MALDI-TOF MS system (MALDI Biotyper) and phenotypic identification system (VITEK2), using $\operatorname{sod} A$ sequencing as the reference standard.

\section{Methods}

\section{Study design and obtained data}

A retrospective, multicenter, case-control study was conducted at the Hiroshima University Hospital, Hiroshima General Hospital, and Hiroshima City Asa Citizens Hospital in Hiroshima, Japan. This study recruited all the patients with SBSEC bacteremia admitted between January 1, 2012, and December 31, 2019, at these hospitals. The patients were identified using the microbiology database of each institution. SBSEC bacteremia was defined based on the isolation of the organism from one or more sets of blood culture bottles. The study protocol was approved by the Institutional Review Boards of each institution. The requirement for written informed consent was waived due to the retrospective study design. The patient data obtained from the medical records included age, sex, comorbidities, surgical histories, sources of bacteremia, whether colonoscopies or echocardiographies were performed during the treatment period, and the 30-day all-cause mortality. If the source of the infection was not identified, the bacteremia was classified as a primary bacteremia.

\section{Identification of SBSEC}

The SBSEC isolates were obtained by processing the blood culture samples in the BacT/ALERT 3D (bioMérieux, Marcy l'Étoile, France) at the Hiroshima University Hospital and Hiroshima City Asa Citizens Hospital. In the Hiroshima General Hospital, the blood culture system that was used between 2012 and 2014 was the BacT/ ALERT 3D, and between 2015 and 2019, the VersaTREK (Thermo Scientific, Westlake, OH). SBSEC was identified using an automated phenotypic identification system VITEK2 Compact (bioMérieux) with a VITEK-2 GP ID card (bioMérieux), according to the recommendations of the manufacturer, at the Hiroshima University Hospital and Hiroshima General Hospital. In the Hiroshima City Asa Citizens Hospital, SBSEC was identified using the Rapid ID32 Strep (bioMérieux). The SBSEC isolates were stored as glycerol stocks at $-70{ }^{\circ} \mathrm{C}$ until use. Species re-identification of SBSEC isolates was performed using sequencing sodA, MALDI-TOF MS using the MALDI Biotyper, and phenotypic identification using the VITEK2 Compact at the Hiroshima University Hospital. For the $\operatorname{sodA}$ sequencing analysis, bacterial DNA was extracted using the QIAamp DNA Blood and Tissue Mini Kit (Qiagen, Germany). The sodA PCR was performed as described previously [16]. The amplicons were sequenced using the ABI Prism 3130xl Genetic Analyzer (Applied Biosystems, Foster City, CA) with a Big-Dye kit (Applied Biosystems). The amplified sequences were compared with reference sequences from the GenBank database using a BLAST search (https://www.ncbi.nlm.nih.gov/ BLAST/). A sequence similarity of $99 \%$ was used as a "cutoff" value for the SBSEC species identification [17].

A MALDI-TOF MS analysis was performed using a BD MALDI Biotyper Sirius system (Becton, Dickinson and Company, USA) with the MBT Compass 4.1 that included the MBT Compass library Ver.9.0.0.0 (8468MSPs) (Bruker Daltonik GmbH, Bremen, Germany). Bacteria were cultured on $5 \%$ sheep blood agar 
(Eiken Chemical, Tokyo, Japan) in $5 \% \mathrm{CO}_{2}$ at $37{ }^{\circ} \mathrm{C}$ for $24 \mathrm{~h}$. A MALDI-TOF MS analysis using pure culture colonies was performed using direct transfer methods, as described previously [18]. As specified by the manufacturer, identification scores $\geq 2.0$ were accepted for reliable identification at the species/subspecies level. The results with the highest scores were reported.

\section{Statistical analysis}

The continuous variables were analyzed using the Wilcoxon rank-sum test. The categorical variables were analyzed using the $\chi^{2}$ test or Fisher's exact test for small samples. All the statistical analyses were performed using JMP 16.0 (SAS Institute Inc., Cary, NC, USA). All the $P$ values were two-sided, and statistical significance was set at $P<0.05$.

\section{Results}

\section{Clinical characteristics of patients with SBSEC}

During the study period, 39 patients with SBSEC bacteremia were identified (Hiroshima University Hospital, 25 cases; Hiroshima General Hospital, nine cases; and Hiroshima City Asa Citizens Hospital, five cases). Of these, SGSP $(n=29,75 \%)$, SGSG $(n=5,13 \%)$, S. lutetiensis (SL, formerly S. infantarius subsp. coli) $(n=4,10 \%)$, and S. infantarius subsp. infantarius (SISI) $(n=1,3 \%)$ were identified using the $\operatorname{sodA}$ sequencing analysis.

The demographic and clinical characteristics of the patients with SBSEC bacteremia are summarized in Table 1 . The median age of the patients was 76 (range 28-92) years. There were 25 men (64\%). One pregnant woman with SGSP bacteremia underwent cesarean section, followed by heart valve replacement surgery. There were no pediatric patients (aged $<18$ years). Primary bacteremia was the most common source of SBSEC bacteremia $(n=14,36 \%)$, followed by infective endocarditis $(n=10,26 \%)$ and biliary tract infections $(n=9,23 \%)$. There were no significant differences in the frequencies of the source of bacteremia among the SBSEC subspecies isolates (only the statistical data of SGSP and SGSG are shown in Table 1). Of all patients, 54\%, 41\%, and $95 \%$ underwent transthoracic electrocardiographies, colonoscopies, and computed tomography (CT) scans, respectively. Overall malignancies were diagnosed in $54 \%$ of patients with SBSEC bacteremia. There were no significant differences in the incidence of overall malignancies among each SBSEC subspecies (data not shown). However, the incidence of overall colorectal cancer and colorectal cancer identified after the diagnosis of bacteremia in patients with SGSG bacteremia was significantly higher than that in patients with SGSP bacteremia $(P=0.048$ and 0.015 , respectively). In addition, the incidence of overall colorectal cancer in patients with
SGSG bacteremia was significantly higher than that in patients with bacteremia due to other SBSEC subspecies $(P=0.049)$. The overall 30 -day mortality rate was $13 \%$ (5 of 39 cases); all five patients had SGSP bacteremia and two of them had polymicrobial bacteremia.

\section{Identification of SBSEC}

The identification results of 39 isolates from patients with SBSEC bacteremia are shown in Table 2. The MALDI Biotyper correctly identified all the SBSEC isolates at the species level and 92\% (36 of 39) at the subspecies level, which was confirmed using $\operatorname{sod} A$ sequencing. In the identification process using MALDI Biotyper, the identification results of all SBSEC isolates ranked 1-10 were SBSEC species. In 19 of 39 isolates, the results of the identification with score $\geq 2.0$ using the MALDI Biotyper included other SBSEC species. The MALDI Biotyper identified all SGSPs at the subspecies level correctly. However, only $80 \%$ of the SGSG and $75 \%$ of the SL isolates were identified correctly at the subspecies level. One isolate each of SGSG and SL were misidentified as SGSP, and one SISI isolate was misidentified as S. equinus.

In contrast, the VITEK2 correctly identified 87\% (34 of 39) of the SBSEC isolates at the species level and 67\% (26 of 39) at subspecies level. The VITEK2 identified all the SGSGs at the subspecies level correctly. However, the VITEK2 identified 20 (69\%) SGSPs with a high probability, five (17\%) with low discrimination, four (14\%) with no identification, and no isolate was misidentified. One SL isolate was misidentified as SGSP by the VITEK2.

The MALDI Biotyper was significantly more accurate in identifying SBSEC isolates at the subspecies level compared to the VITEK2 (92\% vs. $67 \%, P=0.010)$. In contrast, there were no significant differences in the rates of correct identification of SBSEC isolates at the species level between the MALDI Biotyper and the VITEK2 $(100 \%$ vs. $87 \%, P=0.055)$.

\section{Discussion}

In the present study, SGSP (74\%) was the dominant subspecies of SBSEC that caused bacteremia, followed by SGSG (13\%), SL (10\%), and SISI (3\%). These results were consistent with those of previous studies [7, 19, 20]. Specific diseases have been found to be associated with specific SBSEC species or subspecies. For instance, SGSG bacteremia was found to be associated with infective endocarditis and colorectal cancer $[4,5]$. In addition, SGSP and S. infantarius were found to be associated with biliary tract infections and biliary-pancreatic cancer [6-9]. In the present study, excluding primary bacteremia, infective endocarditis was the most common cause of bacteremia (26\%), followed by biliary tract infections (23\%). These results were also consistent with those of 
Table 1 Demographic and clinical characteristics of patients with bacteremia due to Streptococcus bovis/Streptococcus equinus complex

\begin{tabular}{|c|c|c|c|c|c|c|}
\hline Characteristics & $\begin{array}{l}\text { S. gallolyticus subsp. } \\
\text { pasteurianus }(n=29)\end{array}$ & $\begin{array}{l}\text { S. gallolyticus } \\
\text { subsp. } \\
\text { gallolyticus } \\
(n=5)\end{array}$ & S. lutetiensis $(n=4)$ & $\begin{array}{l}\text { S. infantarius } \\
\text { subsp. } \\
\text { infantarius } \\
(n=1)\end{array}$ & $\begin{array}{l}\text { Total } \\
(n=39)\end{array}$ & $\begin{array}{l}P \text { value } \\
\text { SGSP vs. SGSG }\end{array}$ \\
\hline Age, median (range) & $74(28-92)$ & $81(69-83)$ & $82(77-92)$ & 91 & $76(28-92)$ & 0.39 \\
\hline Gender, men (\%) & $18(62)$ & $4(80)$ & $3(75)$ & $0(0)$ & $25(64)$ & 0.63 \\
\hline Pregnant women & $1(3)$ & $0(0)$ & $0(0)$ & $0(0)$ & $1(3)$ & 1 \\
\hline \multicolumn{7}{|l|}{ Co-morbidities } \\
\hline Diabetes mellitus & $7(24)$ & $0(0)$ & $1(25)$ & $0(0)$ & $8(21)$ & 0.56 \\
\hline Liver cirrhosis & $2(7)$ & $0(0)$ & $0(0)$ & $0(0)$ & $2(5)$ & 1 \\
\hline Chronic kidney disease & $1(3)$ & $1(20)$ & $1(25)$ & $1(100)$ & $4(10)$ & 0.28 \\
\hline Neutropenia at culture & $3(10)$ & $0(0)$ & $0(0)$ & $0(0)$ & $3(8)$ & 1 \\
\hline $\begin{array}{l}\text { Gastrointestinal surgery within } \\
30 \text { days }\end{array}$ & $0(0)$ & $0(0)$ & $0(0)$ & $0(0)$ & $0(0)$ & 1 \\
\hline \multicolumn{7}{|l|}{ Malignancies } \\
\hline Overall malignancies & $16(55)$ & $4(80)$ & $1(25)$ & $0(0)$ & $21(54)$ & 0.38 \\
\hline \multicolumn{7}{|l|}{ Gastrointestinal tract } \\
\hline Esophageal & $3(10)$ & $0(0)$ & $0(0)$ & $0(0)$ & $3(8)$ & 1 \\
\hline Stomach & $1(3)$ & $1(20)$ & $0(0)$ & $0(0)$ & $2(5)$ & 0.28 \\
\hline Colon/rectum & $4(14)$ & $3(60)$ & $1(25)$ & $0(0)$ & $8(21)$ & 0.048 \\
\hline Bile duct & $3(10)$ & $0(0)$ & $0(0)$ & $0(0)$ & $3(8)$ & 1 \\
\hline Liver & $1(3)$ & $0(0)$ & $0(0)$ & $0(0)$ & $1(3)$ & 1 \\
\hline \multicolumn{7}{|l|}{ Other malignancies } \\
\hline Hematologic & $4(14)$ & $1(20)$ & $0(0)$ & $0(0)$ & $5(13)$ & 1 \\
\hline Brain & $1(3)$ & $0(0)$ & $0(0)$ & $0(0)$ & $1(3)$ & 1 \\
\hline Oral cavity & $3(10)$ & $0(0)$ & $0(0)$ & $0(0)$ & $3(8)$ & 1 \\
\hline Prostate & $2(7)$ & $0(0)$ & $0(0)$ & $0(0)$ & $2(5)$ & 1 \\
\hline Ovary & $2(7)$ & $0(0)$ & $0(0)$ & $0(0)$ & $2(5)$ & 1 \\
\hline Multiple malignancies (> 1) & $7(24)$ & $1(20)$ & $0(0)$ & $0(0)$ & $8(21)$ & 1 \\
\hline Known before the bacteremia & $13(45)$ & $1(20)$ & $1(25)$ & $0(0)$ & $15(38)$ & 0.38 \\
\hline Discovered after the bacteremia & $3(10)$ & $3(60)$ & $0(0)$ & $0(0)$ & $6(15)$ & 0.029 \\
\hline Colon/rectum & $2(7)$ & $3(60)$ & $0(0)$ & $0(0)$ & $5(13)$ & 0.015 \\
\hline Bile duct & $1(3)$ & $0(0)$ & $0(0)$ & $0(0)$ & $1(3)$ & 1 \\
\hline \multicolumn{7}{|l|}{ Colorectal cancer } \\
\hline Known before the bacteremia & $2(7)$ & $0(0)$ & $1(25)$ & $0(0)$ & $3(8)$ & 1 \\
\hline Discovered after the bacteremia & $2(7)$ & $3(60)$ & $0(0)$ & $0(0)$ & $5(13)$ & 0.015 \\
\hline $\begin{array}{l}\text { Endoscopic examination per- } \\
\text { formed following the bacteremia }\end{array}$ & $10(34)$ & $3(60)$ & $2(50)$ & $1(100)$ & $16(41)$ & 0.35 \\
\hline \multicolumn{7}{|l|}{ Source of bacteremia } \\
\hline Primary bacteremia & $10(34)$ & $1(20)$ & $2(50)$ & $1(100)$ & $14(36)$ & 1 \\
\hline Infective endocarditis & $7(24)$ & $2(40)$ & $1(25)$ & $0(0)$ & $10(26)$ & 0.59 \\
\hline Biliary tract infection & $6(21)$ & $2(40)$ & $1(25)$ & $0(0)$ & $9(23)$ & 0.57 \\
\hline Urinary tract infection & $2(7)$ & $0(0)$ & $0(0)$ & $0(0)$ & $2(5)$ & 1 \\
\hline Others & $4(14)$ & $0(0)$ & $0(0)$ & $0(0)$ & $4(10)$ & 1 \\
\hline \multicolumn{7}{|l|}{ Infective endocarditis } \\
\hline $\begin{array}{l}\text { TTE performed following the } \\
\text { bacteremia }\end{array}$ & $16(55)$ & $2(40)$ & $3(75)$ & $0(0)$ & $21(54)$ & 0.65 \\
\hline $\begin{array}{l}\text { TEE performed following the } \\
\text { bacteremia }\end{array}$ & $0(0)$ & $2(40)$ & $1(25)$ & $0(0)$ & $3(8)$ & 0.018 \\
\hline Computed tomography & $27(93)$ & $5(100)$ & $4(100)$ & $1(100)$ & $37(95)$ & 1 \\
\hline Polymicrobial bacteremia & $6(21)$ & $0(0)$ & $1(25)$ & $0(0)$ & $7(18)$ & 0.56 \\
\hline
\end{tabular}


Table 1 (continued)

\begin{tabular}{|c|c|c|c|c|c|c|}
\hline Characteristics & $\begin{array}{l}\text { S. gallolyticus subsp. } \\
\text { pasteurianus }(n=29)\end{array}$ & $\begin{array}{l}\text { S. gallolyticus } \\
\text { subsp. } \\
\text { gallolyticus } \\
(n=5)\end{array}$ & S. lutetiensis $(n=4)$ & $\begin{array}{l}\text { S. infantarius } \\
\text { subsp. } \\
\text { infantarius } \\
(n=1)\end{array}$ & $\begin{array}{l}\text { Total } \\
(n=39)\end{array}$ & $\begin{array}{l}P \text { value } \\
\text { SGSP vs. SGSG }\end{array}$ \\
\hline Escherichia coli & $3(10)$ & $0(0)$ & $0(0)$ & $0(0)$ & $3(8)$ & 1 \\
\hline Klebsiella pneumoniae & $1(3)$ & $0(0)$ & $0(0)$ & $0(0)$ & $1(3)$ & 1 \\
\hline Corynebacterium jeikeium & $1(3)$ & $0(0)$ & $1(25)$ & $0(0)$ & $2(5)$ & 1 \\
\hline Streptococcus pneumoniae & $1(3)$ & $0(0)$ & $0(0)$ & $0(0)$ & $1(3)$ & 1 \\
\hline Thirty-day all-cause mortality & $5(17)$ & $0(0)$ & $0(0)$ & $0(0)$ & $5(13)$ & 1 \\
\hline
\end{tabular}

TEE transesophageal echocardiography; TTE transthoracic echocardiography; SGSP Streptococcus gallolyticus subspecies pasteurianus; SGSG Streptococcus gallolyticus subspecies gallolyticus

Table 2 Comparison of identification results of 39 isolates of SBSEC using the MALDI Biotyper and VITEK2 compact

\begin{tabular}{|c|c|c|c|c|c|c|c|c|c|}
\hline \multirow{2}{*}{$\begin{array}{l}\text { SBSEC } \\
\text { type }\end{array}$} & \multirow{2}{*}{$\begin{array}{l}\text { Reference } \\
(\operatorname{sod} A), \mathrm{n}\end{array}$} & \multicolumn{3}{|c|}{ MALDI Biotyper } & \multicolumn{5}{|l|}{ VITEK2 } \\
\hline & & $\begin{array}{l}\text { Identified } \\
\text { with } \\
\text { highest } \\
{\text { score } a s^{\mathrm{a}}}\end{array}$ & $\begin{array}{l}\text { Correct } \\
\text { identification } \\
\text { to the } \\
\text { subspecies } \\
\text { level }\end{array}$ & $\begin{array}{l}\text { Correct } \\
\text { identification } \\
\text { to the SBSEC } \\
\text { species level }\end{array}$ & $\begin{array}{l}\text { Identified } \\
\text { with high } \\
\text { probability } \\
\text { as }^{\text {b }}\end{array}$ & $\begin{array}{l}\text { Low } \\
\text { discrimination }\end{array}$ & $\begin{array}{l}\text { Not } \\
\text { identified }\end{array}$ & $\begin{array}{l}\text { Correct } \\
\text { identification } \\
\text { to the } \\
\text { subspecies } \\
\text { level }\end{array}$ & $\begin{array}{l}\text { Correct } \\
\text { identification } \\
\text { to the SBSEC } \\
\text { species level }\end{array}$ \\
\hline SGSP & 29 & 29 SGSP & $29(100)$ & $29(100)$ & 20 SGSP & $5(17)^{c}$ & $4(14)$ & $20(69)$ & $24(83)$ \\
\hline SGSG & 5 & $\begin{array}{l}\text { 4 SGSG, } 1 \\
\text { SGSP }\end{array}$ & $4(80)$ & $5(100)$ & 5 SGSG & $0(0)$ & $0(0)$ & $5(100)$ & $5(100)$ \\
\hline$S L$ & 4 & $\begin{array}{l}3 \mathrm{SL}, 1 \\
\text { SGSP }\end{array}$ & $3(75)$ & $4(100)$ & $1 \mathrm{SL}, 1 \mathrm{SGSP}$ & $2(50)^{d}$ & $0(0)$ & $1(25)$ & $4(100)$ \\
\hline SISI & 1 & $1 \mathrm{SE}$ & $0(0)$ & $1(100)$ & None & $1(100)^{e}$ & $0(0)$ & $0(0)$ & $1(100)$ \\
\hline Total & 39 & - & $36(92)^{*}$ & $39(100)^{\dagger}$ & - & $8(21)$ & $4(10)$ & $26(67)^{*}$ & $34(87)^{\dagger}$ \\
\hline
\end{tabular}

SBSEC Streptococcus bovis/equinus complex; SGSP Streptococcus gallolyticus subspecies pasteurianus; SGSG Streptococcus gallolyticus subspecies gallolyticus; SL Streptococcus lutetiensis; SISI Streptococcus infantarius subspecies infantarius; SE Streptococcus equinus

*MALDI Biotyper was significantly more accurate in identifying the SBSEC isolates at the subspecies level compared with the VITEK2 ( $P=0.010)$

${ }^{\dagger}$ There were no significant differences in the rates of correct identification of the SBSEC isolates at the species level between the MALDI Biotyper and the VITEK2 $(P=0.055)$

${ }^{a}$ Identified with the highest score (score $>2.0$ )

${ }^{b}$ Identified with high probability (>95\%)

Details of "Low discrimination" are as follows

c SGSG (50\%)/SGSP (50\%) n=1; SGSG (33\%)/SGSP (33\%)/SISI (33\%) n=1; SL (33\%)/SGSG (33\%)/SGSP (33\%) $n=1$; SL (51\%)/SGSG (49\%) $n=1$; Lactococcus raffinolactis (34\%)/SL (33\%)/SGSG (33\%) $n=1$

${ }^{d}$ SL (51\%)/SGSG (49\%) $n=1$; SL (50\%)/SGSP $(50 \%) n=1$

e SL (50\%)/SISI (50\%) n=1

previous studies $[8,20]$. However, there were no significant differences in the frequencies of the source of bacteremia among the SBSEC subspecies.

In the present study, there were no significant differences in the incidence of overall malignancies among each SBSEC subspecies. However, the incidence of overall colorectal cancer in patients with SGSG bacteremia was significantly higher than that in patients with bacteremia due to other SBSEC subspecies including SGSP. The strong correlation between colorectal cancer and SGSG has been well-established [5]. A systematic review and meta-analysis showed that the risk of acquiring colorectal cancer was lower in patients with SGSP than in those with SGSG at an odds ratio of 7.26, and that the incidence of colorectal cancer in patients with SGSP did not exceed the incidence in general asymptomatic individuals [5].

As many patients with SBSEC bacteremia have been identified as elderly with comorbidities $[19,20]$, it may have been difficult to perform colonoscopies in all the patients. In the present study, the primary bacterial sources in patients with colorectal cancer were infective endocarditis and primary bacteremia, and no patients had biliary tract infections. As the study used a retrospective design and no patients with biliary tract infection underwent colonoscopies, it is uncertain whether 
these patients had colorectal cancer. However, a previous large-cohort study found that colorectal cancer was less common in patients with biliary tract infections [6]. Therefore, in case of SBSEC bacteremia, patients with biliary tract infections may not require colonoscopies. Nevertheless, further studies are required to determine which patients with SBSEC bacteremia have a lower risk of colorectal cancer.

In previous studies, SGSP was associated with biliary pancreatic cancer [6-8]. In the present study, bile duct cancer was detected in three of 29 patients with SGSP bacteremia. Bile duct cancer was not found in patients with bacteremia caused by SBSEC, except in those caused by SGSP. Additionally, although $95 \%$ of the patients underwent CT scans, no patient was found to have pancreatic cancer. In each previous study, since the number of patients with biliary-pancreatic cancer and SGSP bacteremia was small [6-8], further investigation is required to clarify the association between SGSP bacteremia and biliary-pancreatic cancer.

In the present study, four patients with SL bacteremia were identified. The sources of SL bacteremia were infective endocarditis, biliary tract infections, and primary bacteremia. Since the incidence of SL bacteremia is less common than the incidences of SGSG and SGSP, only a few studies have investigated the clinical characteristics of SL bacteremia [19, 20]. Previous studies have reported infective endocarditis and biliary tract infections as sources of SL bacteremia $[19,20]$. The results of the present study were consistent with those of previous studies, suggesting that the clinical presentation of patients with SL bacteremia is similar to that of patients with SGSG and SGSP.

In the present study, SGSP was identified in a pregnant woman with infective endocarditis. A previous study suggested that SGSP was an important pathogen of pregnancy-related infections leading to neonatal meningitis [21].

Further, the MALDI Biotyper was significantly more accurate in identifying SBSEC isolates at the subspecies level compared to the VITEK2. However, there were no significant difference in the rates of correct identification of SBSEC isolates at the species level between the MALDI Biotyper and the VITEK2. The MALDI Biotyper correctly identified all the SGSP isolates at the subspecies level. In 17 SGSP cases (59\%), all the bacterial species identified using the MALDI Biotyper with a score $>2$ were SGSP. Previous studies have also reported that the MALDITOF MS analyses using the MALDI Biotyper identified SGSP accurately [13, 22]. In contrast, the VITEK2 identified $69 \%$ of SGSP isolates at the subspecies level correctly. Eighty percent of the SGSG isolates were identified correctly at the subspecies level using the MALDI Biotyper.
However, in all the SGSG cases, those with scores $\geq 2.0$ obtained using the MALDI Biotyper included SGSP strains. In contrast, the VITEK2 correctly identified all the SGSG isolates with a high probability . The differences in the results of the MALDI Biotyper analysis may have been due to the number of reference strains of SGSP in the current database being greater than that of SGSG (7 strains vs. 4 strains). SL was more correctly identified at the subspecies level with the use of the MALDI Biotyper (75\%) than with the VITEK2 (25\%). SISI was misidentified with the use of the MALDI Biotyper as $S$. equinus. The same misidentification pattern was reported previously [13]. Moreover, the comparative evaluation of the two MALDI-TOF systems-MALDI Biotyper and VITEK MS - with the 16S rRNA and 16S-23S intergenic spacer region sequencing being used as the reference method, revealed several inaccuracies in both of the systems [13]. Additionally, the accurate identification with the MALDI-TOF MS was correlated significantly with the reference database for the target species [23]. Therefore, it is necessary to increase the number of reference spectra in the database for a more accurate identification of the SBSEC subspecies. The present study found that in terms of association between the subspecies and disease, colorectal cancer was associated significantly with SGSG bacteremia, while there were no significant differences in the frequencies of the source of bacteremia among the SBSEC isolates. The MALDI-TOF MS and phenotypic identification systems in the present and previous studies $[13,22]$ identified the SBSEC isolates at the species level accurately, but not at subspecies level. Additionally, it was difficult to identify SBSEC genetically using sequencing target genes such as $\operatorname{sod} A$ in clinical microbiological laboratories as a routine practice. Therefore, when SBSEC is detected in blood culture, it may be reasonable for clinicians to perform echocardiographies and colonoscopies to detect infective endocarditis and colorectal cancer in all patients with SBSEC bacteremia.

This study had some limitations. This was a retrospective study with a small sample size. The initial methods for identifying SBSEC differed between the three hospitals. There was an absence of a gold standard for the species identification of streptococci. Sequencing of $\operatorname{sod} A$ was performed only as an identification reference in the study. Additionally, in the MALDI-TOF MS analysis, we only analyzed the direct transfer method. Given the retrospective design of this study, $41 \%$ and $54 \%$ of the patients had undergone colonoscopy and transthoracic echocardiography, respectively, while only $8 \%$ underwent transesophageal echocardiography. Therefore, infective endocarditis and colorectal cancer may have been underestimated because not all patients underwent echocardiographies and colonoscopies. 
However, to the best of our knowledge, this was the largest study that investigated the incidence of SBSEC bacteremia in Japan. Further large-scale, prospective studies are required to clarify the clinical and microbiological characteristics of SBSEC bacteremia.

\section{Conclusions}

We have reviewed the clinical characteristics of patients with SBSEC bacteremia retrospectively and evaluated the accuracy of identification of SBSEC isolates using the MALDI-TOF MS and phenotypic identification systems, with $\operatorname{sod} A$ gene sequencing as a reference. While the source of bacteremia was similar between each SBSEC subspecies, colorectal cancer was associated significantly with SGSG bacteremia. The MALDI-TOF MS was significantly more accurate in identifying SBSEC isolates at the subspecies level compared with the phenotypic identification systems. The accurate distinction between the SBSEC subspecies using the MALDI-TOF MS and phenotypic identification systems remains difficult. However, the MALDI-TOF MS and phenotypic identification systems can accurately identify SBSEC at the species levels. Therefore, when SBSEC is detected in a blood culture, it may be reasonable for clinicians to perform echocardiography and colonoscopy to investigate infective endocarditis and colorectal cancer.

\begin{abstract}
Abbreviations
SBSEC: Streptococcus bovis/Streptococcus equinus Complex; SGSG: Streptococcus gallolyticus subspecies gallolyticus; SGSP: Streptococcus gallolyticus subspecies pasteurianus; MALDI-TOF MS: Matrix-assisted laser desorption/ionization time-of-flight mass spectrometry; SL: Streptococcus lutetiensis; SISI: Streptococcus infantarius subspecies infantarius.
\end{abstract}

\section{Acknowledgements}

We would like to thank Editage (www.editage.com) for English language editing. A part of this work was performed at the Natural Science Center for Basic Research and Development, Hiroshima University.

\section{Authors' contributions}

HK designed the study. YK, HK, KT, HT, MI, MK, and TH participated in the data acquisition. YK, HK, and KT participated in the data analysis and interpretation. YK and HK drafted the manuscript. TN, KO, NS, ST, and HO revised the manuscript. All the authors read and approved the final manuscript.

\section{Funding}

This research received no specific grant from any funding agency in the public, commercial, or not-for-profit sectors.

\section{Availability of data and materials}

The dataset analyzed in this study is available from the corresponding author upon reasonable request.

\section{Declarations}

\section{Ethics approval and consent to participate}

The study protocol was approved by the Institutional Review boards of Hiroshima University, Hiroshima General Hospital, and Hiroshima City Asa Citizens Hospital. All the procedures were performed in accordance with the Declaration of Helsinki and relevant guidelines. All the data were anonymized, and the requirement for written informed consent was waived by the review boards of Hiroshima University, Hiroshima General Hospital, and Hiroshima City Asa Citizens Hospital due to the retrospective study design.

\section{Consent for publication}

Not applicable.

\section{Competing interests}

The authors declare that they have no competing interests.

\section{Author details}

${ }^{1}$ Department of Surgery, Graduate School of Biomedical and Health Sciences, Hiroshima University, Hiroshima, Japan. ${ }^{2}$ Department of Infectious Diseases, Hiroshima University Hospital, Hiroshima, Japan. ${ }^{3}$ Project Research Center for Nosocomial Infectious Diseases, Hiroshima University, Hiroshima, Japan. ${ }^{4}$ Section of Clinical Laboratory, Division of Clinical Support, Hiroshima University Hospital, Hiroshima, Japan. ${ }^{5}$ Division of Laboratory Medicine, Hiroshima University Hospital, Hiroshima, Japan. ${ }^{6}$ Department of Surgery, JA Hiroshima General Hospital, Hiroshima, Japan. ${ }^{7}$ Present Address: Section of Clinical Research and Laboratory, JA Hiroshima General Hospital, Hiroshima, Japan. ${ }^{8}$ Department of Surgery, Hiroshima City Asa Citizens Hospital, Hiroshima, Japan. ${ }^{9}$ Department of Clinical Laboratory, Hiroshima City Asa Citizens Hospital, Hiroshima, Japan. ${ }^{10}$ Translational Research Center, Hiroshima University, Hiroshima, Japan.

Received: 3 September 2021 Accepted: 15 November 2021

Published online: 26 November 2021

\section{References}

1. Schlegel L, Grimont F, Collins MD, Régnault B, Grimont PA, Bouvet A. Streptococcus infantarius sp. nov., Streptococcus infantarius subsp. infantarius subsp. nov. and Streptococcus infantarius subsp. coli subsp. nov., isolated from humans and food. Int J Syst Evol Microbiol. 2000;50:1425-34. https://doi.org/10.1099/00207713-50-4-1425.

2. Poyart C, Quesne G, Trieu-Cuot P. Taxonomic dissection of the Streptococcus bovis group by analysis of manganese-dependent superoxide dismutase gene (sodA) sequences: reclassification of 'Streptococcus infantarius subsp. coli' as Streptococcus lutetiensis sp. nov. and of Streptococcus bovis biotype 11.2 as Streptococcus pasteurianus sp. nov. Int I Syst Evol Microbiol. 2002;52:1247-55. https://doi.org/10.1099/00207713-52-4-1247.

3. Schlegel L, Grimont F, Ageron E, Grimont PAD, Bouvet A. Reappraisal of the taxonomy of the Streptococcus bovis/Streptococcus equinus complex and related species: description of Streptococcus gallolyticus subsp. gallolyticus subsp. nov., S. gallolyticus subsp. macedonicus subsp. nov. and S. gallolyticus subsp. pasteurianus subsp. nov. Int J Syst Evol Microbiol. 2003;53:631-45. https://doi.org/10.1099/ijs.0.02361-0.

4. Vaska VL, Faoagali JL. Streptococcus bovis bacteraemia: identification within organism complex and association with endocarditis and colonic malignancy. Pathology. 2009;41(2):183-6. https://doi.org/10.1080/00313 020802436816.

5. Boleij A, van Gelder MM, Swinkels DW, Tjalsma H. Clinical importance of Streptococcus gallolyticus infection among colorectal cancer patients: systematic review and meta-analysis. Clin Infect Dis. 2011;53(9):870-8. https://doi.org/10.1093/cid/cir609.

6. Corredoira J, Alonso MP, García-Garrote F, García-Pais MJ, Coira A, Rabuñal $\mathrm{R}$, et al. Streptococcus bovis group and biliary tract infections: an analysis of 51 cases. Clin Microbiol Infect. 2014;20(5):405-9. https://doi.org/10. 1111/1469-0691.12333.

7. Sheng WH, Chuang YC, Teng LJ, Hsueh PR. Bacteraemia due to Streptococcus gallolyticus subspecies pasteurianus is associated with digestive tract malignancies and resistance to macrolides and clindamycin. J Infect. 2014;69(2):145-53. https://doi.org/10.1016/j.jinf.2014.03.010.

8. Marmolin ES, Hartmeyer GN, Christensen JJ, Nielsen XC, Dargis R, Skov $M N$, et al. Bacteremia with the bovis group streptococci: species identification and association with infective endocarditis and with gastrointestinal disease. Diagn Microbiol Infect Dis. 2016;85(2):239-42. https://doi.org/ 10.1016/j.diagmicrobio.2016.02.019.

9. Van Samkar A, Brouwer MC, Pannekoek Y, van der Ende A, van de Beek D. Streptococcus gallolyticus meningitis in adults: report of five cases 
and review of the literature. Clin Microbiol Infect. 2015;21(12):1077-83. https://doi.org/10.1016/.cmi.2015.08.003.

10. Pompilio A, Di Bonaventura G, Gherardi G. An overview on Streptococcus bovis/Streptococcus equinus complex isolates: identification to the species/subspecies level and antibiotic resistance. Int J Mol Sci. 2019;20(3):480. https://doi.org/10.3390/ijms20030480.

11. Hinse D, Vollmer T, Erhard M, Welker M, Moore ER, Kleesiek K, et al. Differentiation of species of the Streptococcus bovis/equinus-complex by MALDI-TOF mass spectrometry in comparison to sodA sequence analyses. Syst Appl Microbiol. 2011;34(1):52-7. https://doi.org/10.1016/j.syapm. 2010.11.010.

12. Romero B, Morosini Ml, Loza E, Rodríguez-Baños M, Navas E, Cantón R, et al. Reidentification of Streptococcus bovis isolates causing bacteremia according to the new taxonomy criteria: still an issue? J Clin Microbiol. 2011;49(9):3228-33. https://doi.org/10.1128/JCM.00524-11.

13. Agergaard CN, Knudsen E, Dargis R, Nielsen XC, Christensen JJ, Justesen US. Species identification of Streptococcus bovis group isolates causing bacteremia: a comparison of two MALDI-TOF MS systems. Diagn Microbiol Infect Dis. 2017;88(1):23-5. https://doi.org/10.1016/j.diagmicrobio. 2017.02.007.

14. Takamura N, Kenzaka T, Minami K, Matsumura M. Infective endocarditis caused by Streptococcus gallolyticus subspecies pasteurianus and colon cancer. BMJ Case Rep. 2014. https://doi.org/10.1136/bcr-2013-203476.

15. Miyahara K, Tobe S, Shizuku T, Inamoto R, Katayama I. Colon cancer of Peutz-Jeghers syndrome with gallolyticus endocarditis. Clin J Gastroenterol. 2020;13(4):517-21. https://doi.org/10.1007/s12328-019-01080-9.

16. Poyart C, Quesne G, Coulon S, Berche P, Trieu-Cuot P. Identification of streptococci to species level by sequencing the gene encoding the manganese-dependent superoxide dismutase. J Clin Microbiol. 1998;36:41-7. https://doi.org/10.1128/JCM.36.1.41-47.1998.

17. López Roa P, Sánchez Carrillo C, Marín M, Romero F, Cercenado E, Bouza E. Value of matrix-assisted laser desorption ionization-time of flight for routine identification of viridans group streptococci causing bloodstream infections. Clin Microbiol Infect. 2013;19(5):438-44. https://doi.org/10. 1111/j.1469-0691.2012.03837.x.

18. Schulthess B, Brodner K, Bloemberg GV, Zbinden R, Böttger EC, Hombach M. Identification of Gram-positive cocci by use of matrix-assisted laser desorption ionization-time of flight mass spectrometry: comparison of different preparation methods and implementation of a practical algorithm for routine diagnostics. J Clin Microbiol. 2013;51:1834-40. https:// doi.org/10.1128/JCM.02654-12.

19. Lazarovitch T, Shango M, Levine M, Brusovansky R, Akins R, Hayakawa K, et al. The relationship between the new taxonomy of Streptococcus bovis and its clonality to colon cancer, endocarditis, and biliary disease. Infection. 2013;41:329-37. https://doi.org/10.1007/s15010-012-0314-x.

20. Ben-Chetrit E, Wiener-Well Y, Kashat L, Yinnon AM, Assous MV. Streptococcus bovis new taxonomy: does subspecies distinction matter? Eur J Clin Microbiol Infect Dis. 2017;36(2):387-93. https://doi.org/10.1007/ s10096-016-2814-6.

21. Li Y, Chen X, Zhang Z, Wang L, Wang J, Zeng J, et al. Microbiological and clinical characteristics of Streptococcus gallolyticus subsp. pasteurianus infection in China. BMC Infect Dis. 2019;19(1):791. https://doi.org/10. 1186/s12879-019-4413-5

22. Gherardi G, Palmieri C, Marini E, Pompilio A, Crocetta V, Di Bonaventura G, et al. Identification, antimicrobial resistance and molecular characterization of the human emerging pathogen Streptococcus gallolyticus subsp. pasteurianus. Diagn Microbiol Infect Dis. 2016;86(4):329-35. https://doi. org/10.1016/j.diagmicrobio.2016.09.019.

23. Seng P, Drancourt M, Gouriet F, La Scola B, Fournier PE, Rolain JM, et al. Ongoing revolution in bacteriology: routine identification of bacteria by matrix-assisted laser desorption ionization time-of-flight mass spectrometry. Clin Infect Dis. 2009;49(4):543-51. https://doi.org/10.1086/600885.

\section{Publisher's Note}

Springer Nature remains neutral with regard to jurisdictional claims in published maps and institutional affiliations.

Ready to submit your research? Choose BMC and benefit from:

- fast, convenient online submission

- thorough peer review by experienced researchers in your field

- rapid publication on acceptance

- support for research data, including large and complex data types

- gold Open Access which fosters wider collaboration and increased citations

- maximum visibility for your research: over $100 \mathrm{M}$ website views per year

At BMC, research is always in progress.

Learn more biomedcentral.com/submissions 